\title{
Study of the propagation of a light beam at the exit of a single-mode and multimode optical fiber
}

\author{
J. C. Natividad; H. H. Cerecedo-Núñez, and P. Padilla-Sosa \\ Laboratorio de Óptica Aplicada, Faculdad de Física de la Univerdad Veracruzana, \\ Zona Universitaria, Xalapa-Enríquez, Veracruz, México.
}

Received 1 May 2020; accepted 8 July 2020

\begin{abstract}
Traditionally, optical fibers have been used as communication lines and optical sensors; however, these have multiple other uses, for example, the interaction and entrapment of microparticles. This article studies the computational modeling of the propagation of light that comes out of conventional, single-mode, and multimode optical fibers, which is of interest when studying the interaction of light with microparticles. As a parameter of analysis and quantification, we use the degree of diffraction of the light propagation beams, at different distances from the optical fibers. Resulted intensity field distributions give us important microscopic information to consider for light interaction with such microparticles.
\end{abstract}

Keywords: Optical fiber; radiation pressure; poynting vector; interaction and trapping of microparticles.

PACS: 42.81.-i; 78.20.Bh; 41.20.-q

DOI: https://doi.org/10.31349/RevMexFisE.18.28

\section{Introduction}

The present research arises from the need to understand the propagation and interaction of a beam at the exit of an optical fiber. For several years the existence of forces produced by the electromagnetic field that comes out of the tip of an optical fiber has been reported [1]. In a laboratory, these forces can be calculated by tracking a particle, to graph its position against time (with respect to some reference frame), as proposed by Alvarado Z. J. C., (2013) [2]; in this way, its equation of motion is obtained, which is derived twice to obtain the acceleration equation. Then we can know the force if we know the mass of the tracked particle. The mentioned method turns out to be indirect and laborious, and the measurements obtained present a lot of uncertainty.

Hernández Zavala J. E., et al. (2014) [3], performed the first simulation in the Computational Fluid Dynamics (CFD) program, where the speed, pressure, and temperature in particles were studied, due to a light beam coming out of the optical fiber, but the results of the simulations do not provide enough information on the fields to study.

Given this situation, it was decided to perform computational simulations and broaden the scene of what happens with the light beam at the exit of the optical fiber. In this way, we will know the characteristics of the forces that occur in the beam at the exit of the fiber without having to measure them directly, only by knowing the parameters of the light and the optical fiber of interest. This will help us to understand better the favorable regions to place a microparticle and thus know the interaction it will suffer. But the study would also help us to observe the behavior of the electric field and the how it propagates in different external media to the optical fiber, as well as the consequences that arise due to the type of optical fiber used. Being more ambitious, we should be able to study the relationship between the conversion of light energy to heat energy.
The contribution of this article is to be able to perform simulations and study the phenomena present in the beam that comes out of the optical fiber since experimentally it is of high difficulty to carry out the measurements directly.

To accomplish our research, we will consider the existing theory in the literature as a theoretical model that describes our propagation beam, and the physical characteristics of the fibers of interest, as well as an existing program to carry out these simulations.

\section{Gaussian beam}

In our study, in a first approximation, the beam at the exit of the optical fiber can be described by the Gaussian beam theory, because when the beam leaves the fiber, according to Carrasco (2014) [4], it does so as a Gaussian beam, see Figs. 1 and 2. Saleh, et al., (1991) [5], consider that this theory allows us to know the opening angle of the beam by performing a mathematical analysis that starts from an electric field $U(\mathbf{r})$. An expression for the complex amplitude $U(\mathbf{r})$ of the Gaussian beam is

$$
\begin{aligned}
U(\mathbf{r}) & =A_{0} \frac{W_{0}}{W(z)} \exp \left(-\frac{\rho^{2}}{W^{2}(z)}\right) \times \cdots \\
& \times \exp \left(-j k z-j k \frac{\rho^{2}}{2 R(z)}+j \zeta(z)\right),
\end{aligned}
$$

defining $\rho^{2}=x^{2}+y^{2}$.

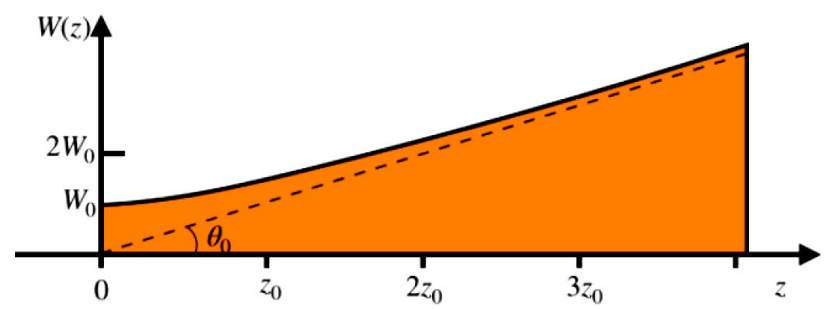

FIGURE 1. Cut in a quadrant, of the cross-sectional representation of a Gaussian Beam. 


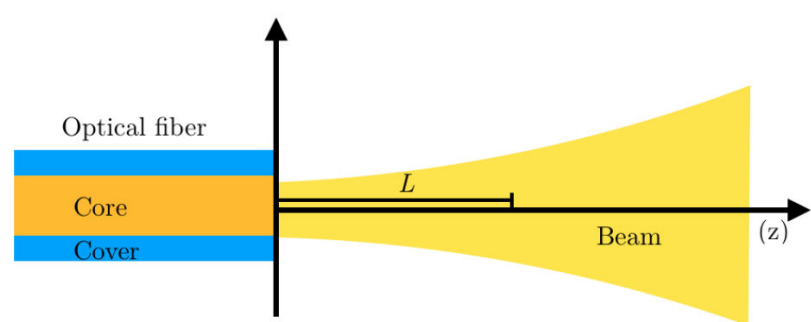

FIGURE 2. Diagram of a beam of light at the exit of an optical fiber.

From this expression, we can study the following parameters

$$
\begin{aligned}
W(z) & =W_{0}\left(1+\left(\frac{z}{z_{0}}\right)^{2}\right)^{\frac{1}{2}} \\
R(z) & =z\left(1+\left(\frac{z_{0}}{z}\right)^{2}\right) \\
\zeta(z) & =\tan ^{-1} \frac{z}{z_{0}} \\
W_{0} & =\left(\frac{\lambda z_{0}}{\pi}\right)^{\frac{1}{2}} \\
\theta_{0} & =\frac{\lambda}{\pi W_{0}}
\end{aligned}
$$

where $W(z)$ is the radius of the beam, $R(z)$ the radius of curvature, $\zeta$ is a phase, $W_{0}$ the radius of the beam's waist, $z_{0}$ is the Rayleigh range, and $\theta_{0}$ the divergence angle. These parameters can be seen in Fig. 1.

As is known, the optical fibers consist of a core and a cladding. The core has a slightly higher refractive index than the cladding, so that, by total internal reflection, the light is confined in the core. The way to measure the divergence angle of the beam coming out of the optical fiber is through the numerical aperture [5], defined as

$$
N A=n \operatorname{sen} \theta_{\max }=\sqrt{n_{n}^{2}-n_{c}^{2}}
$$

where $n$ is the refractive index of the propagation medium, $n_{n}$ is the refractive index of the fiber core, and $n_{c}$ is the refractive index of the cover. So that the divergence angle $\theta_{0}$, of a Gaussian beam, can be related to the angle associated with the numerical aperture $\theta_{\max }$.

\section{Computational simulation}

To perform the computer simulations of the beam's propagation, we used the COMSOL MULTIPHYSICS 5.3a program. This program uses Maxwell's equations for dielectric medium,

$$
\begin{aligned}
\nabla \cdot \vec{D} & =\rho \\
\nabla \cdot \vec{B} & =0 \\
\nabla \times \vec{E} & =-\frac{\partial \vec{B}}{\partial t} \\
\nabla \times \vec{H} & =\vec{J}+\frac{\partial \vec{D}}{\partial t},
\end{aligned}
$$

and, after a mathematical treatment, using boundary conditions for two dielectric materials and the definitions of electric displacement vector and magnetic intensity vector, as well as Ohm's law for homogeneous, isotropic and linear means, it is solved, for any problem, the following equation

$$
\nabla \times\left[\frac{1}{\mu_{r}} \nabla \times \vec{E}\right]-k_{0}^{2}\left[\varepsilon_{r}-\frac{j \sigma}{\omega \varepsilon_{0}}\right]=0 .
$$

This program uses the finite element method, described by Bathe (1986) [8], to solve the Eq. (9).

On the other hand, a geometry that depended on the type of optical fiber (single-mode or multimode) was built. This geometry consists of two rectangles that represent the core and cladding of the fiber, and a third rectangle that simulates the medium where the light propagates at the exit of the optical fiber.

The radius of the nucleus depends on the type of fiber. For the single-mode fiber, a core radius $a=4.1 \mu \mathrm{m}$ was used, a cladding radius of $62.5 \mu \mathrm{m}$, a core refractive index of 1,474, a cladding refractive index of 1,4507 , a propagation power of the light within the $100 \mathrm{~mW}$ optical fiber, a wavelength 980 $\mathrm{nm}$, an initial electric field of $1,194 \times 10^{6} \mathrm{~V} / \mathrm{m}$, and water as a medium of propagation of light at the exit of the fiber (refractive index of 1.3330). Similar parameters were used for multimode fiber, but with the core radius of $a=25.0 \mu \mathrm{m}$, and an initial electric field of $1,958 \times 10^{5} \mathrm{~V} / \mathrm{m}$.

The values of the initial electric field $\overrightarrow{E_{0}}$ were calculated from the definition of time-averaged Poynting vector $\vec{S}$, and its relation to the irradiance. Hence, the following expression was obtained

$$
E_{0}=\sqrt{\frac{2 \mu_{0} c_{0} P}{\pi a^{2}}},
$$

where $\mu_{0}$ is the vacuum permeability, $c_{0}$ is the speed of light in vacuum, $P$ is the power used in the experiment, and $a$ is the radius of the optical fiber's core.

\section{Results}

As it is of interest to know the behavior of the propagation of the light beams after the exit of the optical fiber, our modeling is focused on the two known cases, for single-mode fiber, and a conventional multimode fiber; and we will analyze the results based on the Fresnel number. 

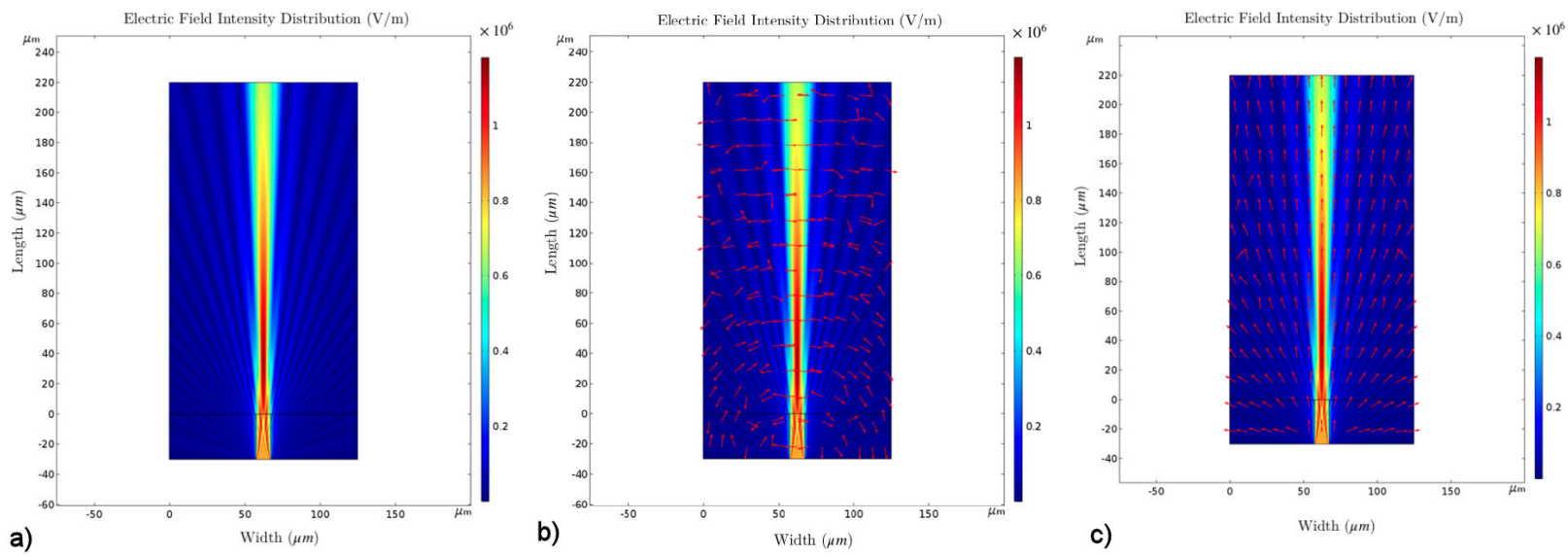

FIGURE 3. Simulation in single-mode fiber. a)Electric field intensity distribution $\vec{E}$. b)Field vectors $\vec{E}$. c)Field vector $\vec{S}$.
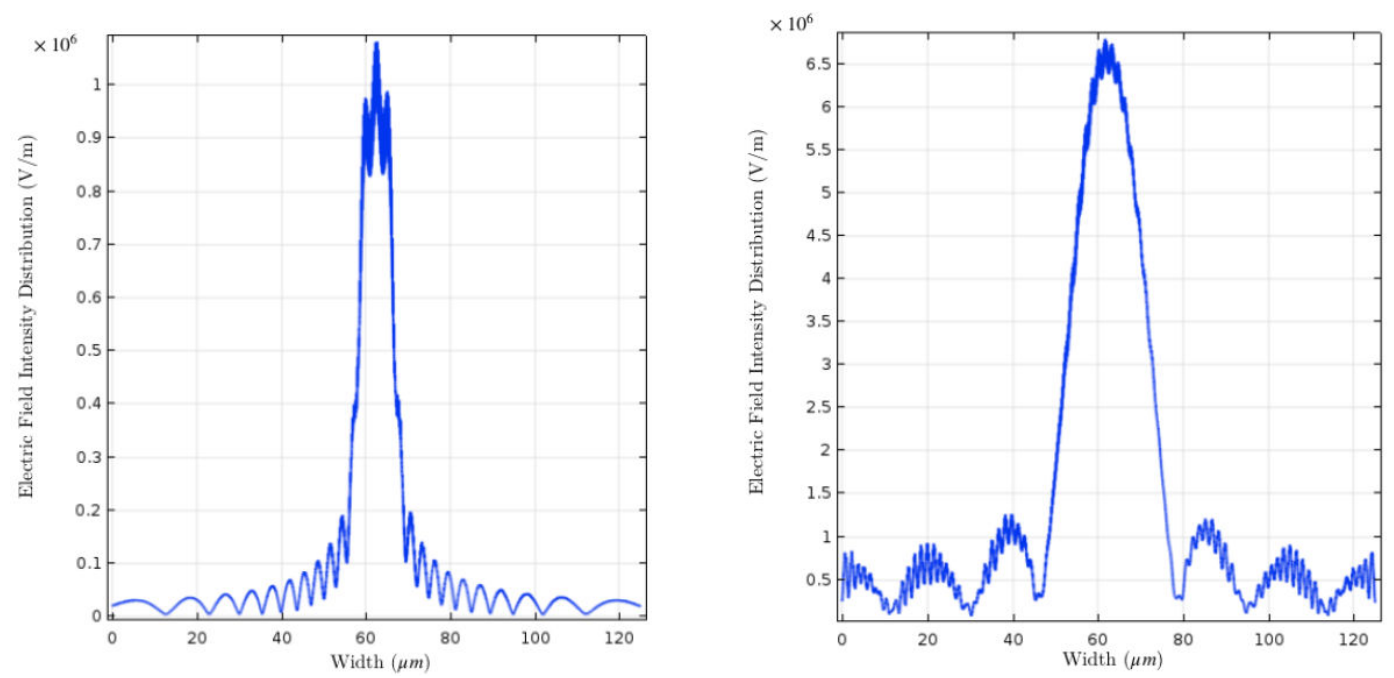

FIGURE 4 . Field intensity profile $\vec{E}$ for a distance $L$ for a single mode fiber tip.

\subsection{Single-Mode Optical Fiber}

The results of the simulation of the single-mode optical fiber are represented in Fig. 3. There, we can see that the intensity of the electric field is not uniform, since it varies in the medium in which it propagates, according to the distance to the tip of the fiber $(L)$ and it is distributed throughout the space; likewise, this field, $\vec{E}$, is more intense at the exit of the fiber up to approximately $80 \mu \mathrm{m}$ away. Figure $3 \mathrm{a}$ ) contains the electric field vectors $\vec{E}$ in the optical fiber simulation. The electric field with and initial direction in $x$ is presented in the expected direction within the core of the fiber, according to the Eq. (3), but outside of it, the vectors change their direction, and even, present changes in their phase. As we move away from the tip of the fiber, we observe that their direction approximates that of a spherical wavefront. The change in the phase of these vectors is due to the diffraction phenomenon. Figure 3c) represents the Poynting vectors $\vec{S}$, which indicate that the energy propagates along the $z$ axis.

The intensity profiles of the electric field, perpendicular to the propagation axis, for different distances $(L)$ to the fiber tip, are analyzed below. It can be seen that some diffraction patterns are depending on the distance to the tip of the fiber. The closer you are to the tip of the fiber, Fresnel diffraction is observed; as $L$ increases, we can see that the diffraction changes to Fraunhofer. The Fig. 4a) represents an intensity profile with Fresnel diffraction; Fig. 4b) expresses an intensity profile with Fraunhofer diffraction.

An important issue is then to corroborate this with the Fresnel number $(N F)$, defined as

$$
N F=\frac{a^{2}}{\lambda L}
$$

where $a$ is the radius of the fiber core, $\lambda$ the wavelength and $L$ is the distance to the tip of the fiber. In the graph of Fig. 5, we can see that most of the diffraction is of the Fraunhofer type (blue zone) and for $L \leq 18 \mu \mathrm{m}$ the diffraction is of Fresnel (orange zone) with the criterion that Fresnel diffraction is presented for $N F \leq 0.1$. The white zone represents the diffraction transition from Fresnel to Fraunhofer.

Similarly, an intensity profile was made along the beam's propagation axis, which is presented in Fig. 6. There, we 


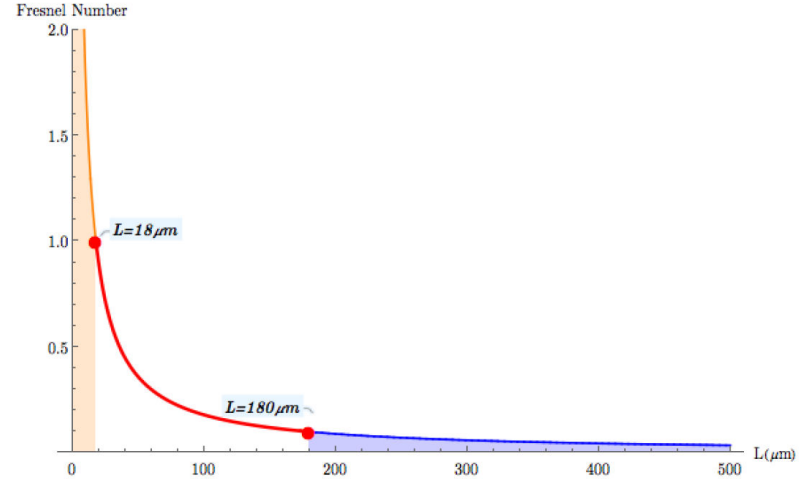

FIGURE 5. Fresnel number as a function of $L$ asociated with single-mode fiber.

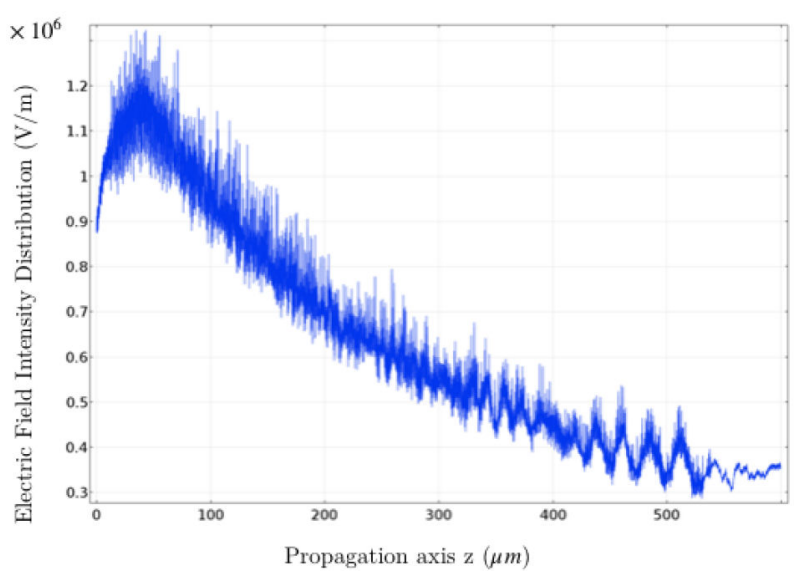

FIGURE 6. Intensity profile propagation of $\vec{E}$ along the propagation axis $(z)$ for a single-mode fiber.
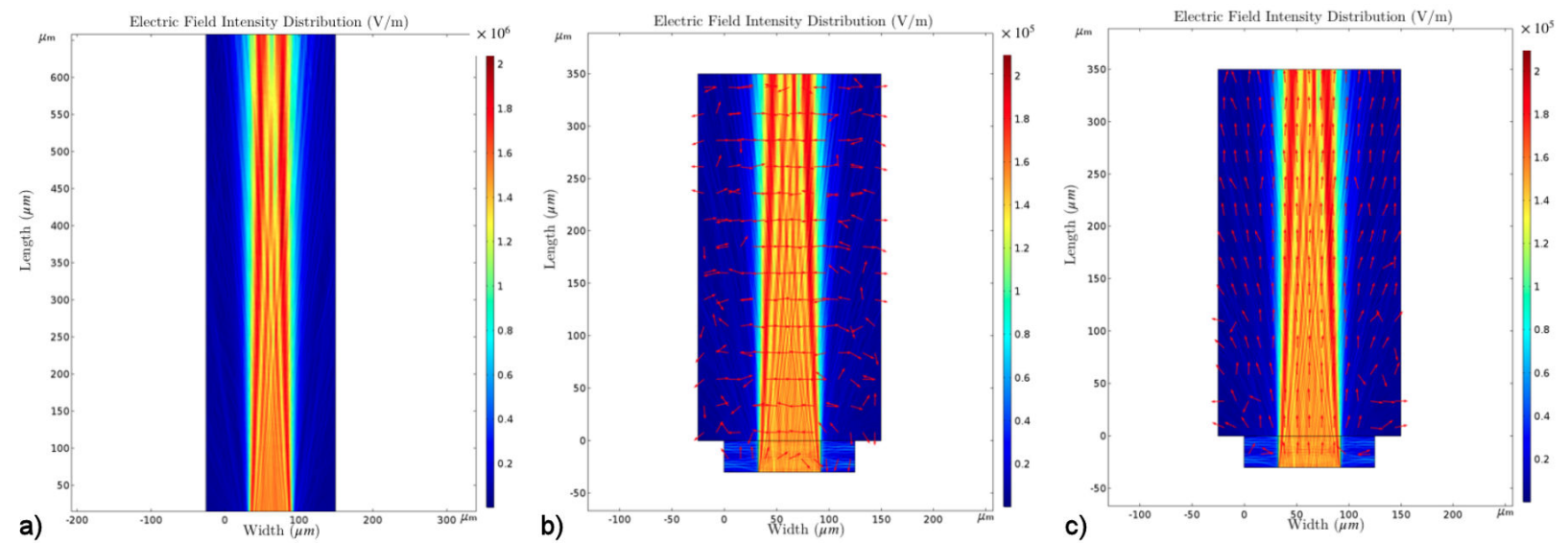

FIGURE 7. Simulation in multimode fiber. a)Electric field intensity distribution $\vec{E}$. b)Field vectors $\vec{E}$. c)Field vectors $\vec{S}$.
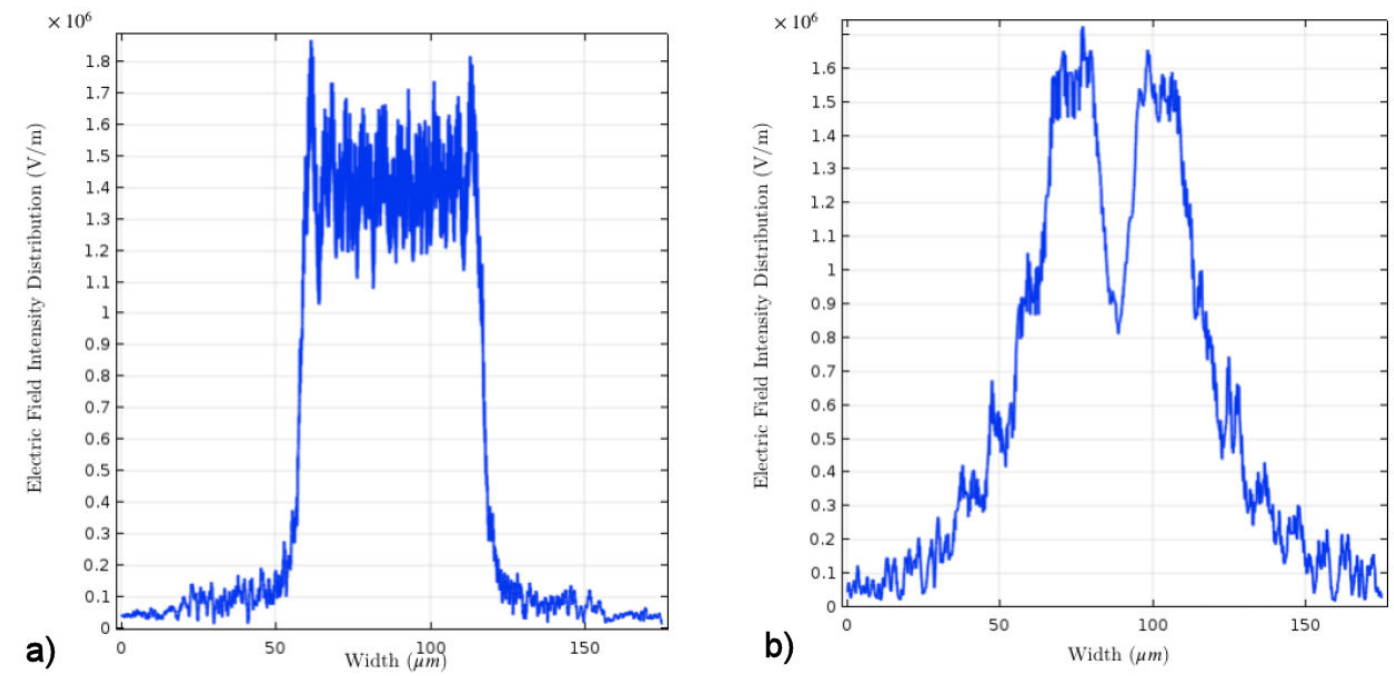

FIGURE 8 . Field intensity profile $\vec{E}$ for a distance $L$ from a multimode fiber tip. a) $L=5 \mu \mathrm{m}$. b) $L=690 \mu \mathrm{m}$.

can see that the the greatest intensity of the electric field is not present exactly at the tip of the optical fiber, but about 10 $\mu \mathrm{m}$ of it. It is very interesting to note also, how the intensity of $\vec{E}$ decays very rapidly as the distance in the propagation axis increases, and it is observed that the greater the distance to the fiber tip $(L)$, the greater the perception of interference, which serves to justify the oscillations at the right end of this graph. 


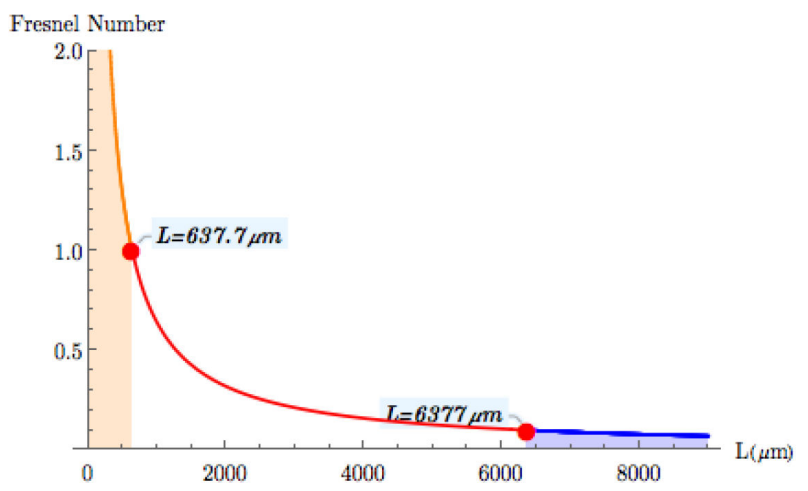

FIGURE 9. Fresnel number as a function of $L$ associated with multimode fiber.

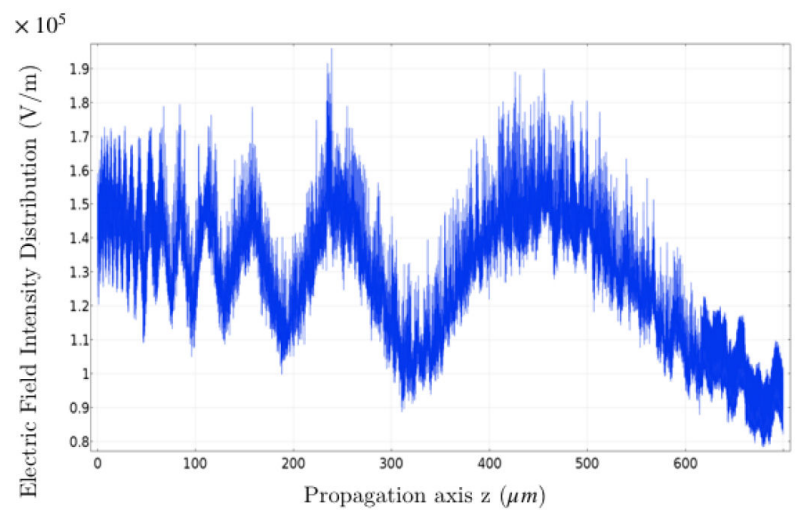

FIGURE 10 . Intensity profile propagation of $\vec{E}$ along the propagation axis $(z)$ for multimode fiber.

\subsection{Multimode Optical Fiber}

In the simulation of Fig. 7a), we can see that the greatest intensity of the light beam converges a few micrometers after the tip of the fiber. This beam is completely different from that of the single-mode fiber, because, along the direction of propagation, it has areas where it becomes less intense and then increases its intensity again. After converging, it begins to diverge, and its intensity begins to decrease.

Figure 8 shows the intensity profiles of the electric field. It is observed that there are diffraction patterns, as in the previous fiber. In the graph of Fig. 8a) $(L=5 \mu \mathrm{m})$, a Fresnel diffraction pattern is shown. This is because this profile corresponds to a distance $\mathrm{L}$ near the tip of the fiber. In Fig. 8b) ( $L=699 \mu \mathrm{m}$ ), we can see that the intensity profile behavior tends to be of the Fraunhofer type.

The graph of Fig. 9, with the Fresnel number, helps us make a comparison of what was obtained in the simulation with what was expected. In this case, the transition between Fresnel and Fraunhofer diffraction is slower than that of single-mode fiber. The orange region of the graph represents the Fresnel diffraction, and the Fraunhofer diffraction is represented by the blue region. Fresnel diffraction is present from small distances to very large distances $(L=637.7 \mu \mathrm{m})$ compared to the previous fiber, and Fraunhofer diffraction starts up to regions of $\mathrm{L}$ greater than $L=6377 \mu \mathrm{m}$.

The graph in Fig. 10 shows an intensity profile of the multimode fiber along the propagation axis. It can be seen that the intensity distribution of the electric field effectively varies along the axis of propagation, and at approximately

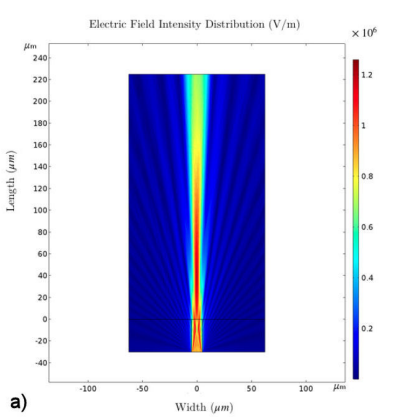

a)

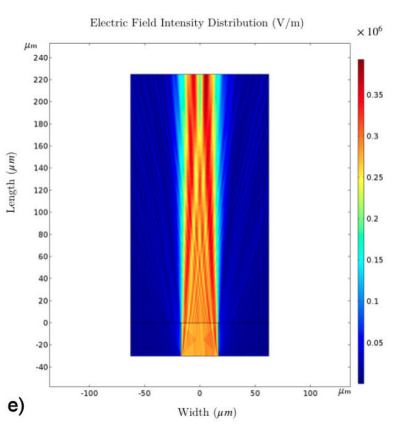

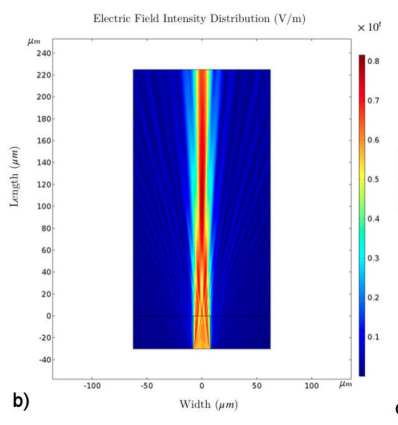

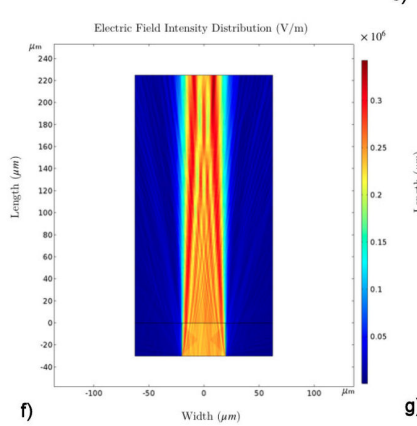

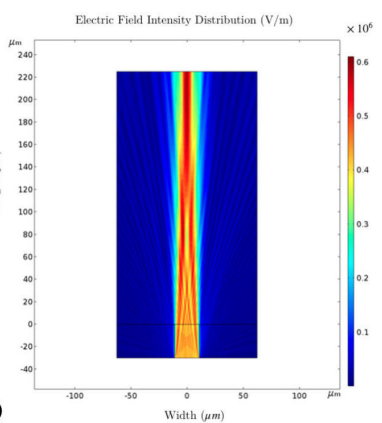

c)

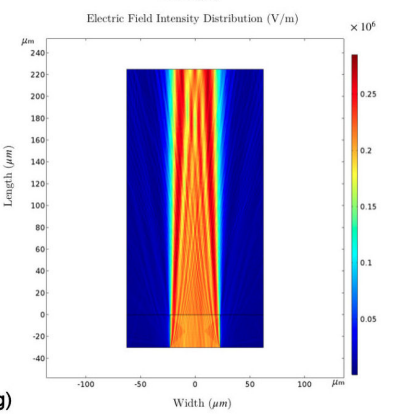

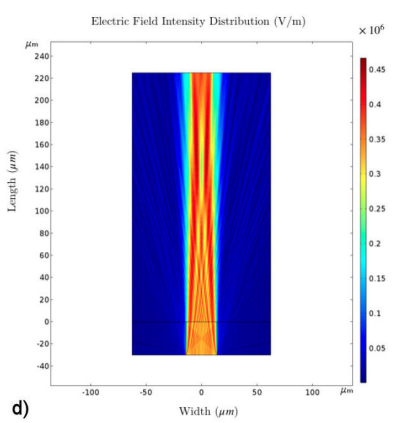

d)

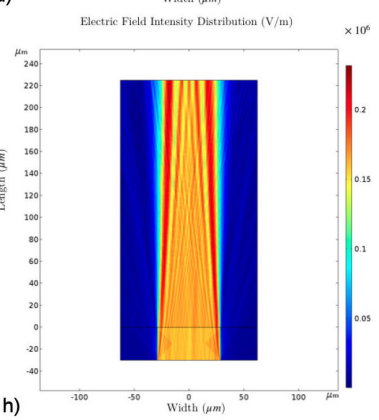

FIgURE 11. Study of a transition from a single-mode to a multimode optical fiber. a) $a=10 \mu \mathrm{m} ; N A=0.14$. b) $a=16 \mu \mathrm{m} ; N A=0.15$ c) $a=22 \mu \mathrm{m} ; N A=0.16 \mathrm{~d}) a=28 \mu \mathrm{m} ; N A=0.17$. e) $a=34 \mu \mathrm{m} ; N A=0.18$. f) $a=40 \mu \mathrm{m} ; N A=0.19 . \mathrm{g}) a=46 \mu \mathrm{m} ; N A=0.20$ h) $a=58 \mu \mathrm{m} ; N A=0.22$ 


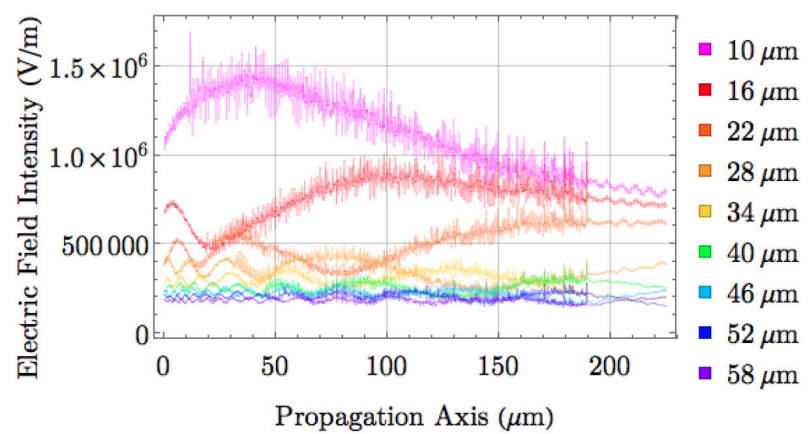

FIGURE 12 . Intensity profile of $\vec{E}$ along the propagation axis $(z)$ for different core diameters.

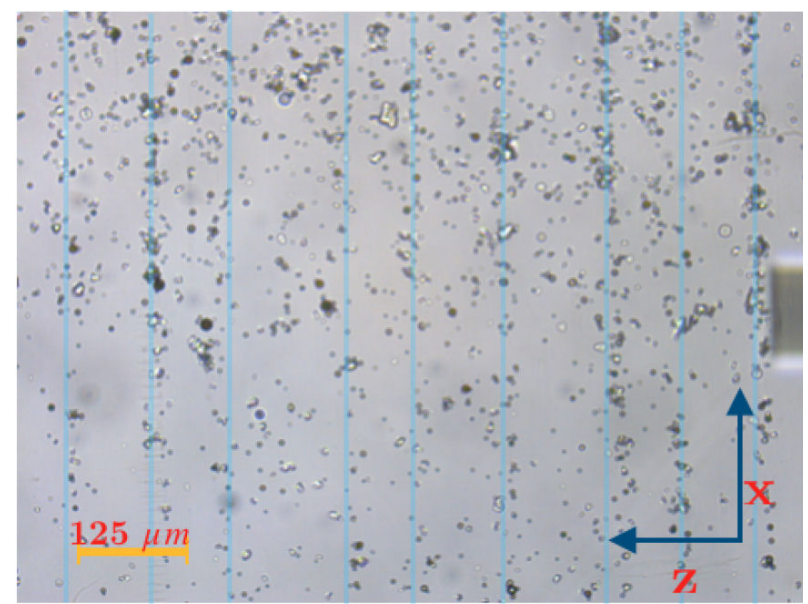

FIGURE 13. Dielectric particles arranged in a periodic distribution, product of the propagation of a beam of light at the exit of a multimode optical fiber (Villegas S. G. (2019) [7].

$450 \mu \mathrm{m}$, the intensity decreases by one order of magnitude and then increases back to the original order. So, it is suggested that this area could be useful for particle entrapment since the particle could be pushed into a potential well.

\subsection{The transition from single-mode to multimode fiber}

In this research we also performed a simulation of the intensity distribution that could be obtained during the transition from single-mode fibers to multi-mode fibers. For this, a visualization was made in the transition from a single-mode fiber to a multi-mode fiber. Its purpose was to observe what happened in the intermediate between these fibers. Although optical fibers with these characteristics are not manufactured, due to the needs of the market.

Figure 11, represents the intensity of the electric field, varying the diameter of the nucleus and the refractive indices of the latter, with the purpose of varying the numerical aperture of N.A. $=0.13$ to N.A. $=0.22$; these data were provided by the commercial provider ThorLabs [9], to satisfy Eq. (7). It can be seen that the distribution of the electric field intensity, in the single-mode multimode transition, the greatest intensity of the electric field distribution also varies. It moves away from the tip of the fiber as the diameter of the core increases. In Fig. 11a), the highest intensity of the electric field is at the exit of the fiber. In Fig. 11b) the intensity moves away from the tip of the fiber, and we can see that the type of distribution observed right after the exit of the fiber is no longer uniform, but it rather presents structures corresponding to Fresnel diffraction. This is presented in the following figures until it ends in Fig. 11h) where the structure presented is a multimode fiber. With this, it can be suggested that the behavior of the beam at the exit of the fiber is varying, and the greatest intensity of the electric field is moving away from it as the diameter of the core increases, leaving behind intensity distributions that correspond to Fresnel diffraction .

In Fig. 12, we can see a comparison of the intensity profiles along the propagation axis for different core diameters. You can see that the intensity of the electric field is lower as the fiber core width increases. This is due to two reasons:

1. The electric field decreases as the area of the core's cross-section increases. This is consequence of the definition of the electric field, described by the Eq. (10).

2. The greatest intensity of the electric field moves away from the tip of the optical fiber as the diameter of the core increases.

Another interesting aspect to observe is the presence of peaks and valleys in the intensity of the electric field $\vec{E}$, which increases until reaching a maximum intensity, and after this, they decline.

It is suggested that the presence of these valleys is related to experimental data obtained by Villegas S. G. (2019) [7], where, after the light beam has interacted with the environment, strips are formed perpendicular to the propagation axis, with a higher concentration of dielectric particles. This may be due to the presence of temperature gradients, caused by the intensity of the light beam in those areas. This accumulation of particles can be seen in Fig. 13.

It should be mentioned that the propagation of light at the exit of the optical fibers was compared with a simulation previously reported with the computational results of Ribeiro R. et al. (2015) [10] and a concordance was found.

Likewise, the variations of the intensity profiles are in agreement with some experimental results that have been observed experimentally with Alvarado Z. J. (2013) [2] and Villegas S. G. (2015) [6].

\section{Conclusions}

In this study, it was possible to simulate the propagation of light at the exit of conventional optical fibers (single-mode and multimode). Studies of intensity profiles were carried out in which it was found that the light beam at the exit of the fiber presents diffraction, as predicted by the fundamental theory. These studies suggest that the mentioned light beam behaves like a beam that passes through a circular opening. The variation of the shape of the beam at the exit of the fiber 
was also simulated when the radius of the core and the numerical aperture of the fiber core are varied.

Regarding intensity distributions in single-mode and multimode fibers, it is possible that the distributions are not uniform, especially in multimode fibers, because of the existence of various propagation modes. In single-mode fibers, light is propagated just in one way, compared to that of multimode fibers, in which multiple modes of propagation are possible; therefore the distribution is more uniform at the exit of the fiber tip compared to multimode, and in the latter, there are multiple modes of propagation. In a single-mode fiber, the greatest intensity of the electric field is right after the exit of the fiber. This does not happen with multimode fiber, as the intensity varies along the axis of propagation.
On the other hand, electric field vectors behave approximately as a spherical wavefront in conventional fibers. The diffraction present in the intensity profiles behaves according to the Fresnel number. In both cases, the diffraction tends to be a Fraunhofer one. An interesting fact is the existence of "electric field intensity wells" in simulated fibers, except in single-mode fiber. It is suggested that these areas could be used for confining microparticles whose dimensions are of just a few micrometers.

As future work, it remains to simulate and study in-depth the force fields acting at the exit of the optical fiber, and also study the relationship between the conversion of light energy to heat energy.
1. A. Ashkin, J. M. Dziedzic, J. E. Bjorkholm, and S. Chu, Optics Letter. 2 (1986) 3. Observation of a single-beam gradient force optical trap for dielectric particles. https://doi. org/10.1364/OL.11.000288

2. J. C. Alvarado Zacarías,. Atrapamiento Óptico utilizando un modelo de trampa con fibras ópticas. Facultad de Física. Xalapa, Ver. Universidad Veracruzana. (Tesis de Licenciatura 2013).

3. J. E. Hernández Zavala, H. H. Cerecedo Nuñez, M.O. Vigueras Zúñiga, P. Padilla Sosa, Proceedings of SPIE. Volume 9164, Article ID 10.1117/12.2086699, 9 pages (2014). Optical Trapping and Optical Micromanipulation XI. https://doi.org/ $10.1117 / 12.2086699$

4. L. Carrasco Fadanelli, Cálculo numérico del efecto de la presión de radiación en micro partículas aplicado al caso de una fibra óptica., Facultad de Física. Xalapa, Ver. Universidad Veracruzana. Tesis de Licenciatura (2014).
5. B. E. Saleh, and M. C. Teich, Fundamentals of photonics Vol. 22 (New York: Wiley 1991).

6. G. Villegas Sánchez, Puntas adelgazadas de fibra óptica como generadores de microburbujas., Facultad de Física. Xalapa, Ver., Universidad Veracruzana. Tesis de Licenciatura (2015).

7. G. Villegas Sánchez, Técnicas de Visión Artificial en la Interacción de Micropartículas con Fibra Óptica., Centro de Investigaciones en Inteligencia Artificial. Xalapa, Ver., Universidad Veracruzana. (2019) Project of PhD in progress. (2018-2022).

8. K. J. Bathe,Finite element procedures. (Doctoral dissertation, Ph. D. Thesis) (Prentice-Hall; 1996).

9. Single mode fiber. Thorlabs (2019). Single mode fiber. Recuperado de https://www.thorlabs.com

10. R. S. R. Ribeiro, O. Soppera, A. G. Oliva, A. Guerreiro, y P. A. Jorge, New trends on optical fiber tweezers. Journal of Lightwave Technology, 33 (2015) 3394-3405. https: // doi.org/10.1109/JLT.2015.2448119 